(C) [2009] IEEE. Reprinted, with permission, from [Ranjit A. Thuraisingham, Yvonne Tran, Ashley Craig, Nirupama Wijesuriya and Hung Nguyen, Using microstate intensity for the analysis of spontaneous EEG:Tracking changes from alert to the fatigue state, Engineering in Medicine and Biology Society, 2009. EMBC 2009. Annual International Conference of the IEEE, 3-6 Sept. 2009]. This material is posted here with permission of the IEEE. Such ermission of the IEEE does not in any way imply IEEE endorsement of any of the University of Technology, Sydney's products or services. Internal or personal use of this material is permitted. However, permission to reprint/republish this material for advertising or promotional purposes or for creating new collective works for resale or redistribution must be obtained from the IEEE by writing to pubs-permissions@ieee.org. By choosing to view this document, you agree to all provisions of the copyright laws protecting it 


\title{
Using microstate intensity for the analysis of spontaneous EEG: Tracking changes from alert to the fatigue state
}

\author{
Ranjit A. Thuraisingham, Yvonne Tran, Ashley Craig, Nirupama Wijesuriya and Hung Nguyen, \\ Senior Member, IEEE
}

\begin{abstract}
Fatigue is a negative symptom of many illnesses and also has major implications for road safety. This paper presents results using a method called microstate segmentation (MSS). It was used to distinguish changes from an alert to a fatigue state. The results show a significant increase in MSS instantaneous amplitude during the fatigue state. Plotting the linear gradient of the nonlinear part of the phase data from the MSS also showed a significant difference $(P<0.01)$ in the gradients of the alert state compared to the fatigue state. The results suggest that MSS can be used in analyzing spontaneous EEG signals to detect changes in physiological states. The results have implications for countermeasures used in detecting fatigue.
\end{abstract}

\section{INTRODUCTION}

$\mathrm{F}$ atigue is a symptom of many acute and chronic illnesses as well as being associated with the "wear and tear" of normal everyday healthy functioning [1]. It can be defined as a state that involves psychological/mental and physical tiredness or exhaustion with a vast range of symptoms including tired eyes, yawning, increased blinking, poor concentration, and low motivation. Fatigue also has major implications for road safety as it has been identified as a major cause of road accidents worldwide and is believed to account for up to $40 \%$ of road crashes [2]. Fatigue related driving accidents are thought to be a result of a decrement in performance of the driver caused by the reduction in his/her level of arousal [3]. To understand this phenomenon there have been studies that have investigated the association between brain electrical activity using electroencephalography (EEG), and the onset of fatigue symptoms. In the EEG, an increased level of alpha activity is often associated with decreased cortical arousal and an

Manuscript received April 6, 2009. This work was supported in part by the Australian Research Council (ARC) Linkage Granting Scheme under Grant LP0560590.

Y. Tran is with the Key University Research Centre in Health Technologies, Faculty of Engineering and Information Technology, University of Technology, Sydney (phone: 612-9514-5555; fax: 303-5555555; e-mail: Yvonne.Tran@uts.edu.au).

R. A. Thuraisingham, is consultant for the Rehabilitation Studies Unit, University of Sydney, (e-mail: ranjit@optusnet.com.au).

A. Craig and N. Wijesuriya are with the Rehabilitation Studies Unit, University of Sydney, (e-mail: a.craig@med.usyd.edu.au and nwij@mail.usyd.edu.au)

H. T. Nguyen is with the Faculty of Engineering and Information Technology, University of Technology, Sydney (email: Hung.Nguyen@uts.edu.au) increase in beta activity has been associated with increased cortical arousal [4]. Thus the majority of studies that have investigated changes in spectral activity during fatigue, have found, for instance, an increase of low frequency EEG waves such as delta $(0.5-3.5)$, theta $(4-7.5 \mathrm{~Hz})$ and alpha $(8-$ $13 \mathrm{~Hz}$ ), while higher frequency waves such as beta rhythms (14-32Hz) will decrease [5].

A novel approach to understanding how brain function changes as a result of decreased arousal levels during fatigue states is to study the microstate segmentations of brain activity. The algorithm used in this paper is based on the technique of microstate segmentation of brain activity $[6,7]$, defined as a functional or physiological brain state during which specific neural computations are performed. It is characterised by fixed spatial distributions of active neuronal generators with time varying intensities [6].

In this paper we analyzed the temporal properties of spontaneous brain activity during two different arousal or physiological states (alert versus fatigue). The EEG recordings consisted of time varying measurements of scalp electric potential field and reflect the dynamics of the functional state of the brain. It was hypothesized that the topographic time course of the scalp electric field can be segmented into stable scalp maps called microstates. The observation is thus expressed by a finite set of microstates, where each time $t$ corresponds to a microstate $k$ with an associated coefficient $a_{k t}$. This coefficient reflects the polarity and strength of the neuronal generators which gives rise to this microstate. It is referred [6,7] to as the microstate intensity. The main feature of this paper was the examination of the use of microstate intensity in EEG analysis. Instead of dealing with multi-channel data, we have a one dimensional time series of $\left\{a_{k t}\right\}$. The focus in segmentation analysis has been to classify the multi-channel observation to certain microstates [6]. In this paper however, the emphasis was on the use of one dimensional microstate intensity time series $\left\{a_{k t}\right\}$ to obtain information about the observed EEG data. We examined one aspect of this series, which was the analysis of the instantaneous amplitude and phase of the envelope time series of $\left\{a_{k t}\right\}$.

In EEG electric potential variations occur due to neuronal firing of the brain. Neurons generate small electrical variation which summed over a region provides the potential variation in that space. This is measured at the various scalp electrodes. In addition, this variation is also measured as a function of time. Instead of analyzing the 
scalp electrode data in terms of wave shapes, the dynamics used in this analysis was based on microstates. Although this approach was originally designed for electrical activity performed on particular tasks called Event Related Potentials (ERP), it can also be applied to spontaneous EEG [6]. In ERP one expects microstates to be more stable during particular tasks, unlike that found in spontaneous EEG. Therefore, instead of examining the noisy structure of the microstate distribution, we focused on the analysis of the time series $\left\{a_{k t}\right\}$.The particular feature we study is the instantaneous amplitude and phase of the envelope time series of $\left\{a_{k t}\right\}$. This is carried out using the analytic signal of $\left\{a_{k t}\right\}$ obtained via the Hilbert transform.

\section{METHODS}

\section{A. Participants}

Fifty participants were randomly selected from a community population to participate in the study. Data from eleven participants could not be used due to impedance problems in one or more electrodes leading to loss of EEG data. Only 39 participants remained and the mean age of this group was 31.1 years (S.D. $=12.3$ years), ranging between 18 and 55 years. Subjects were stratified for sex in order to balance the number of males and female participants in the study. The final number of subjects consisted of 20 females and 19 males. Participants were only admitted into the study if they were currently healthy (as determined by a structured interview before the study), held a current drivers license and reported no prior brain disease or injury. The study was approved by the institutional research ethics committee and participants were only entered into the study after informed consent.

\section{B. Experimental Procedure and EEG Analysis}

In order to create a change in arousal levels leading to fatigue, the experiment condition consisted of assessing vigilance using a cognitive-motor vigilance task called the Divided Attention Steering Simulator (DASS) (Stowood Scientific Instruments). Throughout the task, physiological signals and participants' faces were videoed while performing the simulated driving so that information on when subjects fatigued would be known. EEG signals were also simultaneously taken to measure the neurophysiological changes associated with fatigue.

Participants were instructed to 'drive' in the centre of the road (shown on the computer screen) till they showed definite signs of fatigue. The task was considered monotonous because the participants were required to drive at slow speeds (around 40-60km/hr) for an extended period of time in a noise, stimulus and temperature controlled laboratory. At the same time as driving, vigilance and performance was measured from a reaction time task whereby they were required to respond to a target number that appeared in any of the four corners of the computer screen at random times. Participation in the task was terminated by the researcher when participants showed definite signs of fatigue such as (i) fatigue related facial symptoms such as nodding and prolonged eye closure or (ii) when they deviated off the road for more than $15 \mathrm{~s}$.

EEG within the first 5 minutes of starting the driving task was selected as "alert" EEG and another selection was taken within the 5 minute interval where participants were deemed as fatigued before the task was terminated. Thirtytwo channel EEG data from the 39 participants was analyzed using the microstate segmentation technique. The EEG data was preprocessed so that is was free of artifact. Artifact was removed using a program which employs second-order blind identification (SOBI) and canonical correlation. Two EEG segments, each of 20 s duration, were taken from the alert and fatigue states and combined for the microsegmentation analysis to be carried out on the composite record.

\section{Microstate segmentation model (MSS)}

The electrical activity of the brain can be viewed as a sequence of non-overlapping states with variable duration and intensity. The scalp data in this picture is then represented in terms of a set of normalized vectors $T$, which are the different microstates. At each time $t$, the multichannel data is considered to belong to a microstate $T_{k}$ with a particular intensity which is stored in a matrix A [6].

Suppose $V_{t}$ is a $N_{s} \mathrm{x} 1$ vector consisting of measured scalp potential at the $N_{s}$ scalp electrodes at time $t\left(t=1,2, \cdots N_{T}\right)$. Further, let $T_{k}$ be the normalized $N_{s} \mathrm{x} 1$ vector that represents the $k^{\text {th }}$ microstate and $a_{k t}$ be the intensity at time $t$. The microstate model for the average reference multi-channel data is then represented as:

$$
V_{t}=\sum_{k=1}^{N_{\mu}} a_{k t} T_{k}
$$

where $N_{\mu}$ is the number of microstates. In order to allow for non-overlapping microstates at each time instant, all $a_{k t}$ must be zero except one. Thus at each time instant, the summation in (1) reduces to one non-zero term, corresponding to a single active microstate.

The parameters required to estimate the model are $a_{k t}, T_{k}$ and $N_{\mu}$. The method adopted in this paper is the same basic $N$ microstate algorithm described in [6]. For a given $N_{\mu}$, the process is started with $L_{t} t=1, \ldots N_{T}$, which are the microstate labels associated with each $V_{t}$. We then compute:

$$
S_{k}=\sum_{t \in L_{t}=k}^{N_{T}} V_{t} * V_{t}^{\prime}
$$

The summation here includes only the time points where $L_{t}=k$. The normalized microstate $T_{k}$ is then obtained as the normalized eigenvector corresponding to the largest Eigen value. In equation (2), $V_{t}$ is the transpose of $V_{t}$. The microstate $T_{k}$ associated with the observation $V_{t}$ is the value of $T_{k}$ which minimizes

$$
d_{k t}{ }^{2}=V_{t}^{\prime} * V_{t}-\left(V_{t}^{\prime} * T_{k}\right)^{2}
$$


Thus, a new set of microstate labels are obtained. We then evaluate the function

$$
\sigma_{\mu}^{2}=\sum_{t=1}^{N_{T}}\left(V_{t}^{\prime} * V_{t}-\left(T_{k}^{\prime} * V_{t}\right)^{2}\right) /\left(N_{T}\left(N_{s}-1\right)\right)
$$

The process is iterated till successive values of (4) differ negligibly. For example, in this paper we use 10(-4).

Once convergence is attained for a $\operatorname{particular}_{N_{\mu}}$, we calculate

$\sigma^{2}=\sigma_{\mu}^{2}\left[\left(N_{s}-1\right)^{-1}\left(N_{s}-1-N_{\mu}\right)\right]^{-2}$

$N_{\mu}$ is then estimated by minimizing $\sigma^{2}$.

Once $N_{\mu}$ is estimated, the intensity $a_{k t}$ of the microstate $T_{k}$ which is associated with $V_{t}$ for that $N_{\mu}$ is obtained from

$$
a_{k t}=V_{t}^{\prime} * T_{k}
$$

\section{Analysis of MSS Intensity $\left\{a_{k t}\right\}$}

The coefficient $a_{k t}$ of the microstate $T_{k}$ is related to the intensity of the neuronal generators responsible for this microstate at time $t$. For each time $t$ we have a value of $a_{k t}$. In this section we examined the amplitude and phase of the envelope of the time series $\left\{a_{k t}\right\}$. This is explained in this section. Consider a real time series $\{x(t)\}$. The analytic signal $y(t)$ is defined by:

$$
y(t)=x(t)+i h(t)
$$

where $h(t)$ is the Hilbert transform of $x(t)$,

$$
h(t)=\frac{1}{\pi} P \int_{-\infty}^{+\infty} \frac{x\left(t^{\prime}\right)}{t^{\prime}-t} d t^{\prime}
$$

Here $P$ is the Cauchy principal value. It can be shown [8], that if

where $R$ is the real part, then

$$
x(t)=R\left[a(t) e^{i \phi(t)}\right]
$$

$$
y(t)=a(t) e^{i \phi(t)}
$$

The instantaneous amplitude and phase of the real time series $\{x(t)\}$ is the amplitude and phase of the analytic signal. The phase of the analytic signal is made continuous using the Matlab routine unwrap, which unwraps the phase to make it continuous across the $\pi$.

\section{RESULTS}

In this study we examined whether the amplitude and phase information from $\left\{a_{k t}\right\}$ of MSS is able to distinguish the two states "alert" and "fatigue". Since the MSS was carried out on the composite record (alert (A) (at 0-20s), then fatigue (F) (at 21-40s)), the same type of microstate will represent both alert and fatigue states. However the number of each type of microstate present in alert and fatigue states will be different. The MSS analysis shows that the microstates necessary for proper representation of the composite EEG data varied from 3 to 5 . The number of each these microstates in the alert and fatigue states varied. These numbers presented no obvious feature in the records analyzed, to discriminate A from F.
The top left plot of Figure 1 shows the instantaneous amplitude $(a(t))$ of $\left\{a_{k t}\right\}$ obtained from a composite EEG record. The top right plot shows the average of $a(t)(\operatorname{avg}(t))$, evaluated at each time $t$ using the previous 4 seconds of $a(t)$. Thus $\operatorname{avg}(t)=$ mean $(\operatorname{amp}(t-4: t))$, where the initial value of $t=4$. The result is shown for increments of $t$ of 1 second, beyond $t=4$. The bottom left shows the phase $\phi(t)$ of $\left\{a_{k t}\right\}$ obtained from EEG records. Although $\phi(t)$ appears to be linear, it has structure. This is seen in the bottom right plot where $r(t)$ is drawn, which is the non linear part of $\phi(t)$. It is given by:

$$
r(t)=\phi(t)-\left(c_{0}+c_{1} t\right)
$$

where $c_{0}, c_{1}$ are the intercept and slope of the linear fit to $\phi(t)$.

The results of Figure 1 show that there is an increase in the amplitude after $20 \mathrm{~s}$, in the fatigue state. Further the non-linear of the phase $(r(t))$ shows a change in the direction of the gradient at $\mathrm{t}=21 \mathrm{~s}$.
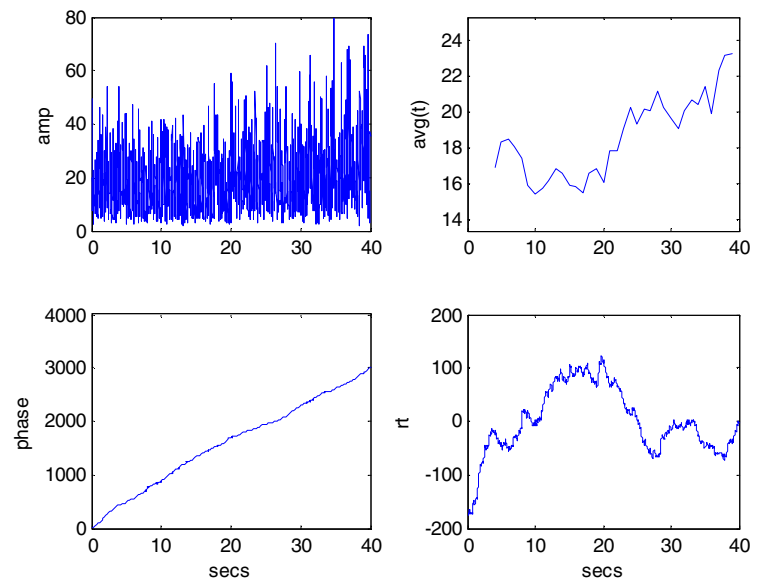

Fig. 1. MSS of spontaneous EEG during alert (0-20s) to fatigue (21-40s) states: The instantaneous amplitude $a(t)$ (Top Left), $\operatorname{avg}(a(t))$ (top right); phase $\phi(t)$ (bottom left); $r(t)$ (bottom right). All plots are drawn as a function of time.

In order to investigate further whether the increase in amplitude in going from the alert to the fatigue state is significant, the mean amplitude in the alert state $(0-20 \mathrm{~s})$ and the mean amplitude in the fatigue state (21-40s) were evaluated. Figure 2 shows a plot of the mean amplitude of the different subjects in the alert state against the difference in the mean amplitude between alert and fatigue. 


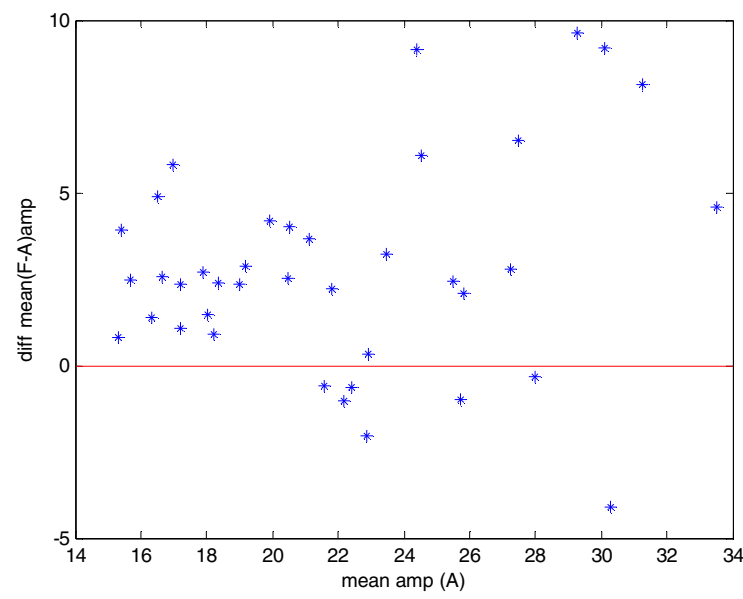

Fig. 2. Plot of the difference in the mean amplitude of (Fatigue-Alert) for different mean amplitudes of alert state. $F=$ Fatigue, $A=$ Alert

The results indicate that in the majority of cases there is an increase in the amplitude in going from alert to fatigue. Dependent t-test analysis revealed significant differences between states $(\mathrm{t}=5.68 ; \mathrm{p}>0.01)$.

Figure 3 examines the linear gradient of the nonlinear part of the phase $(r(t))$ in the alert and fatigue region. Plotted along the $\mathrm{x}$ axis is the gradient of the linear fit of $r(t)$ in the A region(between $t=0$ to $20 \mathrm{~s}$ ). It is denoted by $m(A)$. The values along the y axis are the absolute difference between the gradients of the linear fit of $r(t)$ in the $\mathrm{F}$ region $m(F)$ ( $t=21$ to $40 \mathrm{~s})$ and the A region $m(A)(t=0$ to $20 \mathrm{~s})$. It is denoted by $|m(F)-m(A)|$. The results indicate in general, that there is a difference in the gradient in $r(t)$ in going from A to F. Dependent t-test analysis showed significant differences in the gradients of the two states $(t=7.65 ; \mathrm{p}>0.01)$.

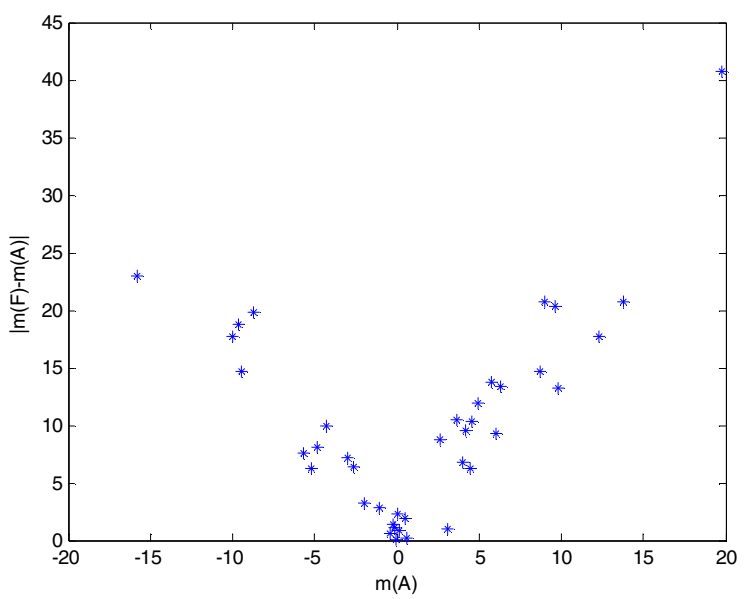

Fig. 3. Plot of the absolute difference in the gradient $|m(F)-m(A)|$ for different gradients of $m(A)$. The gradients are obtained by $r(t)$. F= Fatigue, $\mathrm{A}=$ Alert

\section{CONCLUSION}

The paper examined the use of microstate intensity in analyzing spontaneous EEG data, where the data comprised of recordings of alert and fatigue state in 39 adult participants. The two EEG records were combined and MSS analysis was carried out on the composite record. The focus of this study was to investigate the use of the one dimensional microstate intensity data to analyze the composite record. We examined one aspect of this series, which was the analysis of the instantaneous amplitude and phase of the envelope time series of the microstate intensities. The results indicated that the amplitude and phase of the microstate intensity time series is able to discriminate the two records and provide information on the onset of fatigue in the composite record. The amplitude of the fatigue state was found to be significantly higher than alert. Another study has also examined microstate segmentation in different arousal states such as alert, drowsy, and REM sleep, however, in the alpha band only. They also found that it was drowsiness that showed the greatest amounts of differences in microstates compared to relaxed wakefulness and REM sleep [9]. This method could be implemented into fatigue countermeasures using EEG signals as the success rate of detecting the beginning of fatigue using the change in amplitude was $84 \%$, and $74 \%$ with change in gradient using phase. It was also found that the recordings that detected fatigue from the gradient method were the ones that were difficult to identify when using the amplitude method, hence, the two methods complement each other.

\section{REFERENCES}

[1] J. Shen, J. Barbera and C.M. Shapiro, "Distinguishing sleepiness and fatigue: focus on definition and measurement," Sleep Med. Rev. vol 10, pp. 63-76. 2006

[2] A. Fletcher, K. McCulloch, S.D. Baulk and D. Dawson, "Countermeasures to driver fatigue: a review of public awareness campaigns and legal approaches," Aust. N. Z. J. Public Health vol 29, pp. 471-476. 2005

[3] I. Brown, "Driver Fatigue," Hum. Factors vol 36, pp. 298-314. 1994

[4] Y. Tran, A. Craig and P. McIssac, "Extraversion/introversion and 8$13 \mathrm{~Hz}$ wave in frontal cortical regions," Pers. Individ. Differ. Vol 30, pp. 205-215. 2001

[5] L. J. Trejo, R., Kochavi, K, Kubitz, L. D., Montgomery, R., Rosipal, R., and B. Matthews, "EEG-based estimation of cognitive fatigue." Proceedings of SPIE, vol 5797, pp 105-115. 2005

[6] R. D. Pascual-Marqui, C. M. Michel, and D. Lehmann, "Segmentation of Brain Electrical Activity into Microstates: Model Estimation and Validation", IEEE Transactions on Biomed. Engineering, vol. 7, pp $658-665,1995$.

[7] J. Freudiger "Brain states analysis for direct brain computer analysis" Semester project, Swiss Federal Institute of Technology, June 2003.

[8] W. K. Melville, "Wave modulation and breakdown", J. Fluid Mech (128) 489-506, 1983.

[9] J. L. Cantero, M. Atienza, R. M. Salas, and C. M. Gomez, “ Brain spatial microstates of human spontaneous alpha activity in relaxed wakefulness, drowsiness period, and REM sleep," Brain Topography, vol 11 pp257-263, 1999. 